\title{
A Novel Artificial Intelligent System for Milk Conservation Using Wireless Sensor Networks
}

\author{
M. Sakthivadivel, M. Rathina Priya, N. Krisnaraj and E. Prabhakar
}

\begin{abstract}
The conservation of milk is of major concern in developing countries like India, due to the lack of supply at many household in rural and Industrial areas. In existing system a collection vehicle is assigned for each group of areas. If the vehicle fails to arrive at a particular destination at correct time the milk gets spoiled and becomes useless. The proposed method eliminates this drawback by sensing the quality of milk using $\mathrm{pH}$ meter. The $\mathrm{pH}$ value of good quality milk is 6.7 and higher or lower $p H$ values degrade the quality of milk. This technique directs the vehicle to the appropriate places using alert SMS so that the collection of milk may be fastened up which leads to its ultimate conservation by using wireless sensor networks with induced artificial intelligence.
\end{abstract}

Keywords--- Artificial Intelligence, Alert SMS, Milk Degradation, $p H$ Meter, Wireless Sensor Networks

\section{INTRODUCTION}

$\mathrm{M}$ ILKmay be defined as the whole, fresh, clean, lacteal secretion obtained by complete milking of one or more healthy milch animals (cow or buffalo or goat milk), excluding that obtained within 15 days before or 5 days after calving or such periods as may be necessary to render the milk practically colostrums free and containing the minimum prescribed percentages of milk fat and milk solids not fat (SNF).The price of milk is usually determined by its fat and / or SNF content.[5]The nutrients present in milk are fat, protein, lactose, vitamins and minerals. Other constituents of milk are enzymes, pigments, non-protein nitrogenous substances and gases.

\section{A. Spoilage of Milk}

When raw milk is left standing for a while, it turns "sour". This is the result of fermentation, where lactic acid bacteria ferment the lactose inside the milk into lactic acid.[5] Prolonged fermentation may render the milk unpleasant to

M. Sakthivadivel, Assistant Professor, Department of Information Technology, Dr. Mahalingam College of Engineering and Technology, Pollachi, Coimbatore, India. E-mail: sakthivadivelm@gmail.com

M. Rathina Priya, Assistant Professor, Department of Electronics and Communication Engineering, Dr. Mahalingam College of Engineering and Technology, Pollachi, Coimbatore, India. E-mail: rathinapriyya@gmail.com

N. Krishnaraj, Assistant Professor, Department of Information Technology, Dr. Mahalingam College of Engineering and Technology, Pollachi, Coimbatore, India. E-mail: krishna.rajtce@gmail.com

E. Prabhakar, Assistant Professor, Department of Information Technology, Dr. Mahalingam College of Engineering and Technology, Pollachi, Coimbatore, India. E-mail: prabhakarit10@gmail.com consume.

This fermentation process is exploited by the introduction of bacterial cultures (e.g. Lactobacilli sp., Streptococcus sp., Leuconostoc sp., etc.) to produce a variety of fermented milk products. The reduced $\mathrm{pH}$ from lactic acid accumulation denatures proteins and caused the milk to undergo a variety of different transformations in appearance and texture, ranging from an aggregate to smooth consistency. Some of these products include sour cream, yoghurt, cheese, buttermilk, viili, kefir and kumis. The statistical report of milk consumption during the year 2007-2008 is as follows: In India the total wastage of food is $45 \%$. The annual milk consumption is 100.19 million tons and it is expected to raise 111.12 in 2012.But wastage of milk is $10.2 \%$ approximately 10.11 million tons. The species of the animals which provide milk is reduced to 3/4th of total strength. [5] The population of India is expanding rapidly which results in insufficient supply of milk to many people and milk industries. So there is requirement of a solution to solve this problem.

\section{PROBLEM DESCRIPTION}

Now it is clear that in this current scenario there is the problem that conservation of milk. This problem is because of wastage of milk by spoiling. The milk with high adulteration, bacterial content, less quantity of saturated fat and freshness will get degrade quickly. So there is an issue that lack of supply of milk to rural peoples and milk product industries. No more than 3 levels of headings should be used.

\section{EXISTING SYSTEM}

Figure 1 shows the existing system to collect the milk. The milk is collected from various places are grouped in a collection point where the distributor sales the milk to local people. The remaining milk is used for many industries or by other area people. For collecting the remaining milk a collection vehicle is assigned for each group of areas. After collecting the milk immediate refrigeration process is held in order to avoid it from spoiling. But the problem with this method is due to failure of vehicle to arrive at a correct time to particular destination, the milk gets spoiled. 


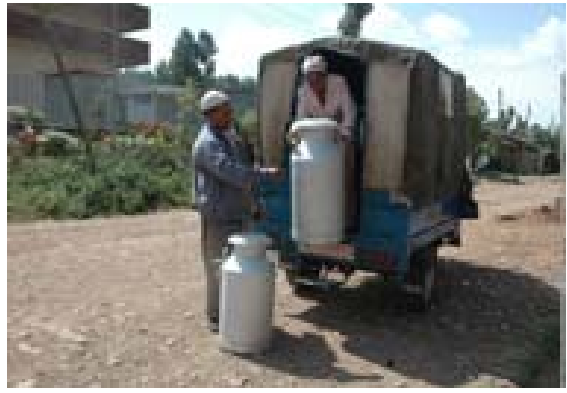

Figure 1: Existing Way of Collecting Milk

\section{PROPOSED MODEL}

This is the modification of the existing system. That is the quality of the milk is determined using their $\mathrm{pH}$ value. To calculate the $\mathrm{pH}$ value a digital $\mathrm{pH}$ meter can be used. Figure 2 shows the digital $\mathrm{pH}$ meter used. The good conditioned milk should have the $\mathrm{pH}$ value as 6.7. If the $\mathrm{pH}$ value of the milk is lower or higher than the required value then it is considered to be the milk which will get spoil quickly. We can eliminate this problem by providing immediate refrigeration to the milk. Normally refrigeration process is held in milk station. Directing the vehicle to the collection point before the milk gets spoiled. So we propose an effective system to achieve this goal which utilizes wireless sensor network with induced artificial intelligence concept.

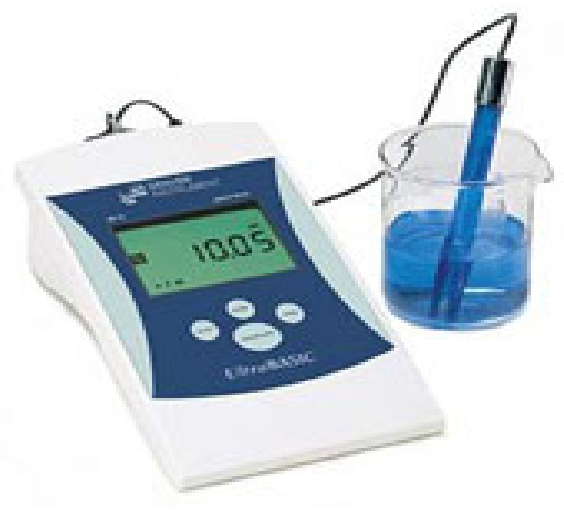

Figure 2: A Digital pH Meter

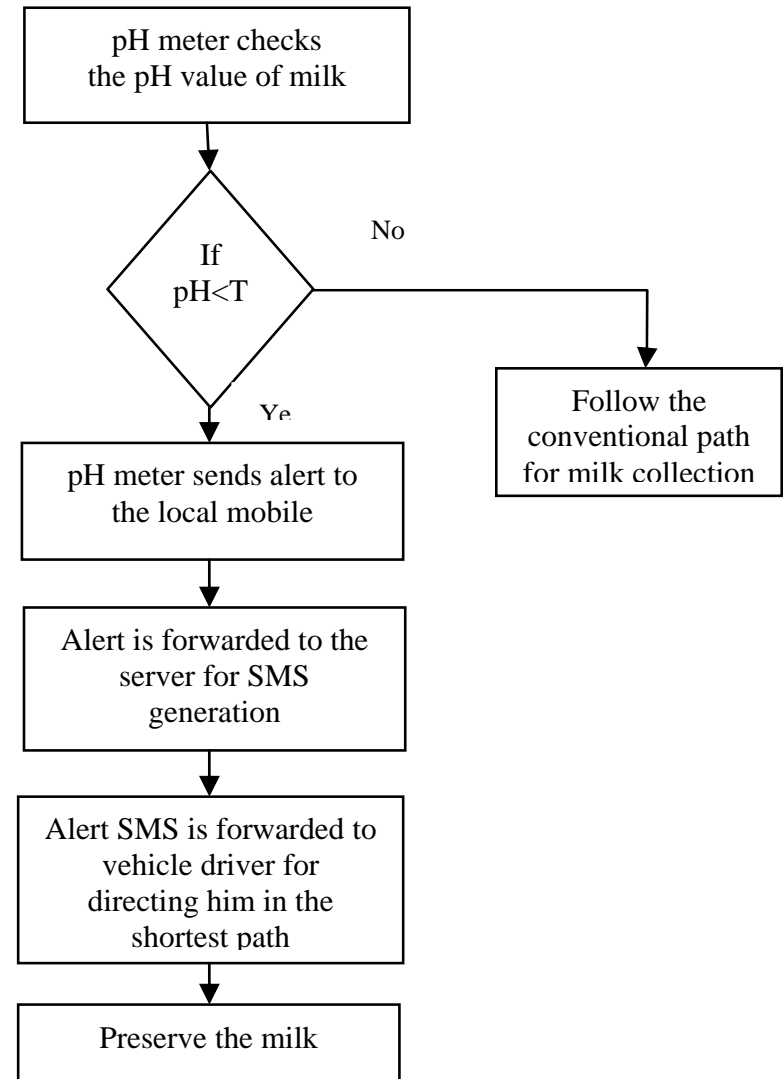

Figure 3: Collection of Milk

\section{SySTEM DESIGN}

This model consist of two sub systems

- Information acquiring system- The information sharing system in the model.

- Alerting system- The system which generates and sends alert to the vehicle driver.

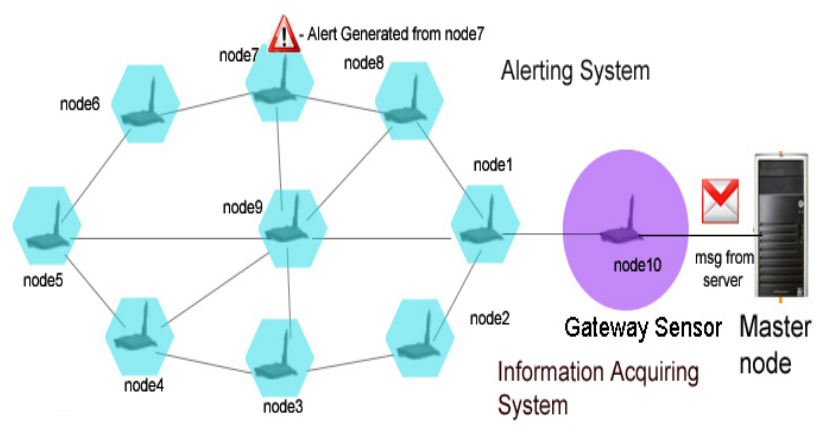

Figure 4: Proposed Model

\section{INFORMATION ACQUIRING SYSTEM}

It is a wireless sensor network which consists of group of sensor nodes with a master node.[6] The components and basics of the wireless sensor network as follows. 


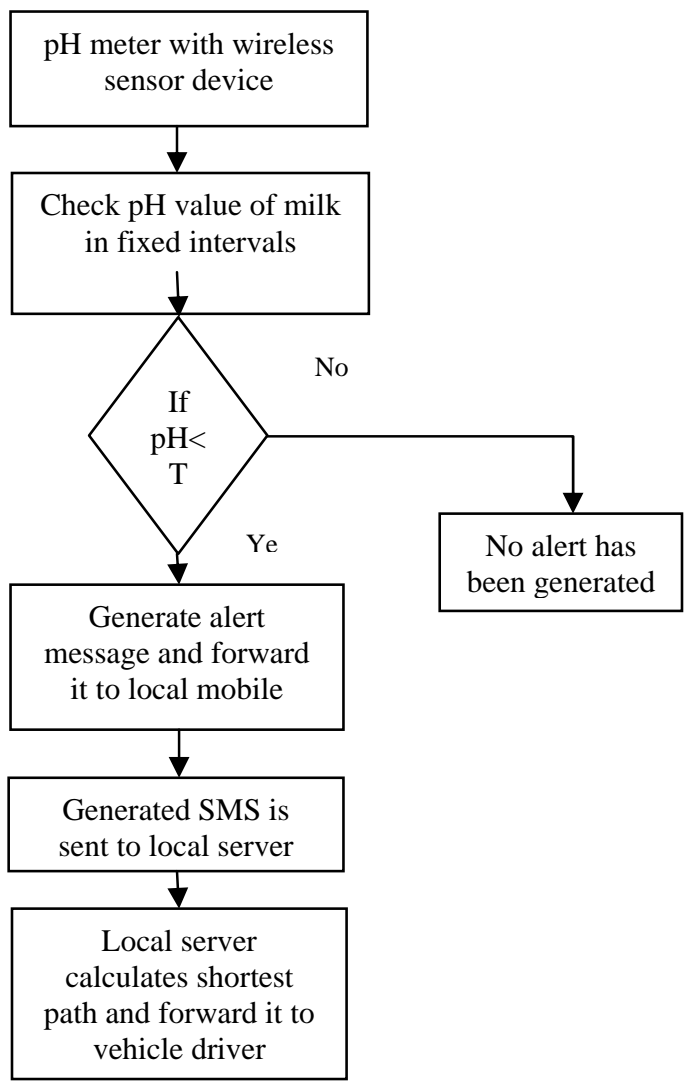

Figure 5: Generation of SMS

\section{A. Wireless Sensor Network}

A wireless sensor network (WSN) is a wireless network consisting of spatially distributed autonomous devices using sensors to cooperatively monitor physical or environmental conditions, such as temperature, sound, vibration, pressure, motion or pollutants, at different locations.[6] Wireless sensor networks are now used in many industrial and civilian application areas, including industrial process monitoring and control, machine health monitoring, environment and habitat monitoring, healthcare applications, home automation, and traffic control[1][2].

\section{B. Sensor Nodes}

Sensor nodes can be imagined as small computers, extremely basic in terms of their interfaces and their components. They usually consist of a processing unit with limited computational power and limited memory, sensors (including specific conditioning circuitry), a communication device (usually radio transceivers or alternatively optical), and a power source usually in the form of a battery.[7] Other possible inclusions are energy harvesting modules, secondary ASICs, and possibly secondary communication devices (e.g. RS-232 or USB).

The base stations are one or more distinguished components of the WSN with much more computational, energy and communication resources. They act as a gateway between sensor nodes and the end user.

The envisaged size of a single sensor node can vary from shoebox-sized nodes down to devices the size of grain of dust.

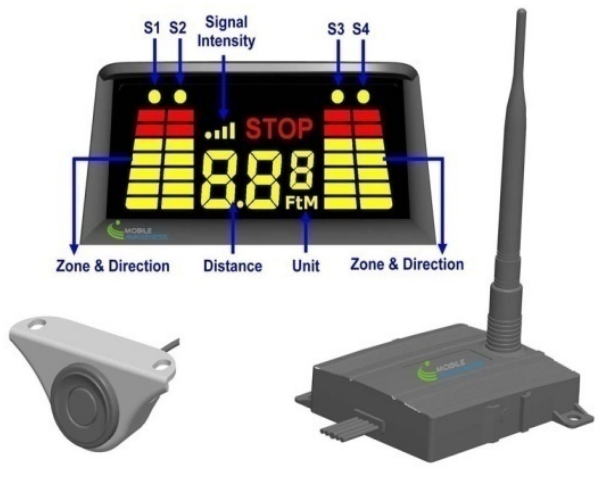

Figure 6: A Typical Digital Sensor Node

The cost of sensor nodes is similarly variable, ranging from hundreds of dollars to a few cents, depending on the size of the sensor network and the complexity required of individual sensor nodes. Size and cost constraints on sensor nodes result in corresponding constraints on resources such as energy, memory, computational speed and bandwidth.[7]A sensor network normally constitutes a wireless ad-hoc network, meaning that each sensor supports a multi-hop routing algorithm (several nodes may forward data packets to the base station).

\section{Platforms Used in WSN}

The following platforms are used in WSN.

\section{i. Standards}

Several standards are currently either ratified or under development for wireless sensor networks[3][4].ZigBee is a mesh-networking standard intended for uses such as embedded sensing, medical data collection, and consumer devices like television remote controls, and home automation. Zigbee is promoted by a large consortium of industry players[9].

\section{ii. Hardware}

The main challenge is to produce low cost and tiny sensor nodes. With respect to these objectives, current sensor nodes are mainly prototypes. Miniaturization and low cost are understood to follow from recent and future progress in the fields of MEMS and NEMS.

\section{iii. Software}

WSNs are meant to be deployed in large numbers in various environments, including remote and hostile regions, with ad-hoc communications as key. For this reason, algorithms and protocols need to address the following issues:

- Lifetime maximization

- Robustness and fault tolerance andSelf-configuration

iv. Operating Systems

Operating systems for wireless sensor network nodes are typically less complex than general-purpose operating systems both because of the special requirements of sensor network applications and because of the resource constraints in sensor network hardware platforms. For example, sensor network applications are usually not interactive in the same way as 
applications for PCs. Because of this, the operating system does not need to include support for user interfaces. Furthermore, the resource constraints in terms of memory and memory mapping hardware support make mechanisms such as virtual memory either unnecessary or impossible to implement.

TinyOS is perhaps the first operating system specifically designed for wireless sensor networks. Unlike most other operating systems, TinyOS is based on an event-driven programming model instead of multithreading. TinyOS programs are composed into event handlers and tasks with run to completion-semantics. When an external event occurs, such as an incoming data packet or a sensor reading, TinyOS calls the appropriate event handler to handle the event.[8] Event handlers can post tasks that are scheduled by the TinyOS kernel some time later. Both the TinyOS system and programs written for TinyOS are written in a special programming language called nesC which is an extension to the $\mathrm{C}$ programming language. NesC is designed to detect race conditions between tasks and event handlers.

\section{v. Algorithms}

WSNs are composed of a large number of sensor nodes; therefore, an algorithm for a WSN is implicitly a distributed algorithm. In WSNs the scarcest resource is energy, and one of the most energy-expensive operations is data transmission. For this reason, algorithmic research in WSN mostly focuses on the study and design of energy aware algorithms for data transmission from the sensor nodes to the base stations. Data transmission is usually multi-hop (from node to node, towards the base stations), due to the polynomial growth in the energycost of radio transmission with respect to the transmission distance.

The algorithmic approach to WSN differentiates itself from the protocol approach by the fact that the mathematical models used are more abstract, more general, but sometimes less realistic than the models used for protocol design.

\section{Functionality of the Information Acquiring System}

For each area a sensor node is placed to monitor. The stepwise algorithm followed by each sensor node in each milk collection unit is as follows:

1. First the digital $\mathrm{pH}$ meter reads the $\mathrm{pH}$ value of the milk in that area.

2. Then, the digital scan sensor reads the $\mathrm{pH}$ value as the sensing value.

3. Then it sends the value to the master node.

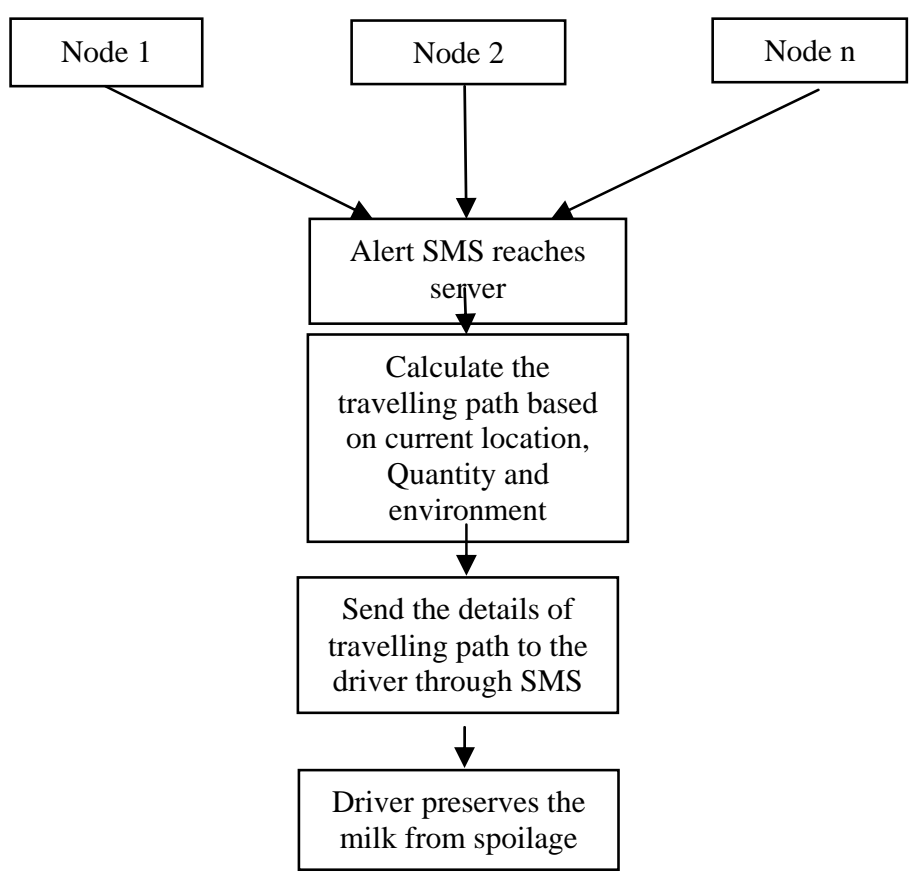

Figure 7: Adhoc Collection of Milk

\section{ALERTING SYSTEM}

The alerting system is the one which generate an alert to the vehicle driver through a Short Message Service (SMS). This system uses the artificial intelligence concept. So there is no need for human resources. In this system an artificial intelligent program is running which reads the percept $(\mathrm{pH}$ value of milk in each area) from all sensor nodes. This program also uses an efficient shortest path finding algorithm (e.g. OSPF) to find the shortest path among all the areas which specifies the low or high $\mathrm{pH}$ value.

\section{A. Functionalities of the Alerting System}

1. First collects the information from various nodes.

2. It is given to the Server Program

3. If the $\mathrm{pH}$ values from various nodes have the critical values (low or high), then by routing algorithm it finds the shortest path.

4. The program generates an SMS alert to the vehicle driver's phone number.

5. Returns the path to the vehicle driver.

\section{IMPLEMENTATION}

The system is implemented by using the Tiny Os and platform independent java language.

\section{A. Tiny O Sin Nesc}

There are two types of components in nes C: modules and configurations. An example program given in Appendix-I shows the simple test program "Blink" which causes the red LED to turn on and off at $1 \mathrm{~Hz}$.

\section{B. A Sample Java Code to Send an SMS}

This Java sample program to send SMS from your PC using GSM modem connected to your computer to your COM 
port.

This module includes the following java files to function.

1. SerialConnection.java (This file is used to connect to your COM port from java program)

2. SerialConnectionException.java (This file is for handling serial connection exceptions in the Java program)

3. SerialParameters.java (This module is used to set your COM port properties for connecting to the com port from the java program)

4. Sender.java (This is the module that implements runnable and sends SMS using the serial connection)

5. SMSClient.java (This java class is the main class that can be instantiated in the java program and called to send SMS. This program in turn will use all the above four files internally to send out your SMS).

The sample code is given in Appendix-II.

\section{COST BeNEFITS ANALYSIS}

Here is the estimated analysis on cost. These values are approximate values and based on the weather condition and milk collection unit size the cost savings may increase well.

- Cost for purchasing digital pH meter- Rs. 800

- Cost for sensor node Rs. 1500

- Maintenance and power consumption cost per year Rs. 500

- Total investment per milk collection unit- Rs. 2800

- Wastage cost per day per milk collection unit

o The probability of wastage is $10 \%$

o Amount of milk gets wasted minimum 10 liters per week.

o So the cost of wastage is $10 * 20=$ Rs. 200

o Per year wastage Rs. 10400

- $\quad$ The minimum gain is Rs. 7600

\section{FutURE ENHANCEMENT}

Vehicle tracking can be effectively implemented by employing the open shortest path algorithm along with additional parameters like the current position of the vehicle, the possible arrival time to the destination and the priority in case of two or more milk collection point sends the SMS alert and climatic condition like rain to conserve milk effectively.

\section{Simulation}

The following snap shots show how the artificial intelligent system conserves milk effectively.

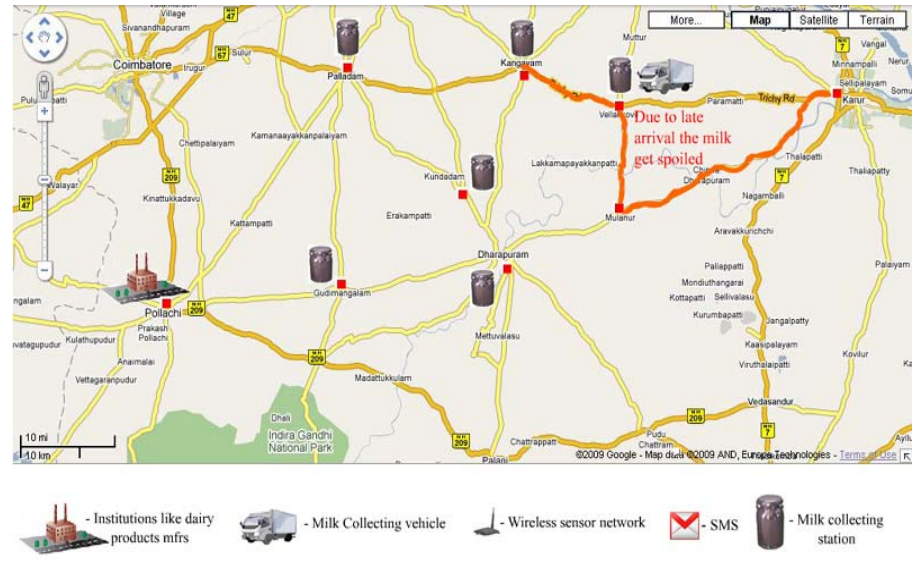

Figure 8: Spoilage of Milk Due to Late Arrival

From figure 8, it is clear that if the vehicle travels in a predefined path, at some stations, the milk may get spoiled because of late arrival. The driver does not know that the milk is going to spoil soon at those stations.

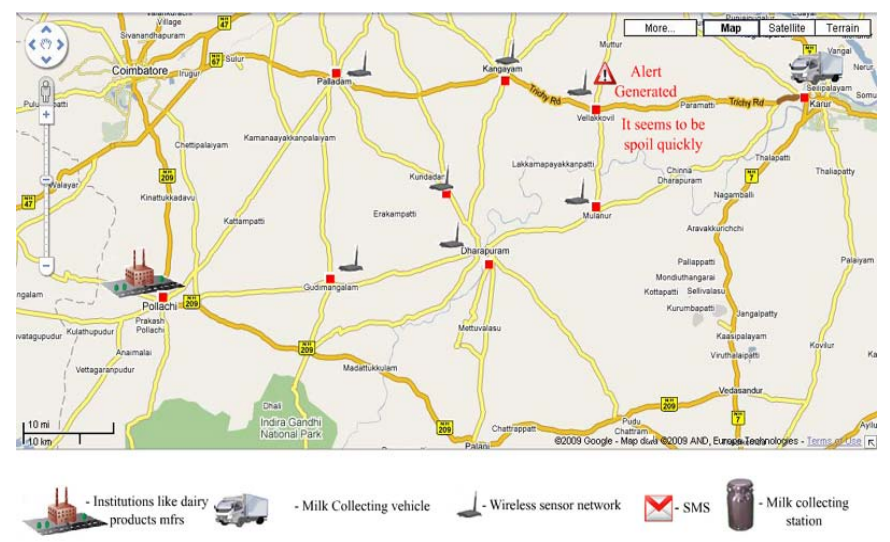

Figure 9: Generation of Alert Regarding the Spoilage of Milk

The $\mathrm{pH}$ meter tests the $\mathrm{pH}$ value of the milk and automatically generates an alert to the local server about the spoilage of milk if the vehicle reaches the destination in predefined path. The server generates an alert SMS.

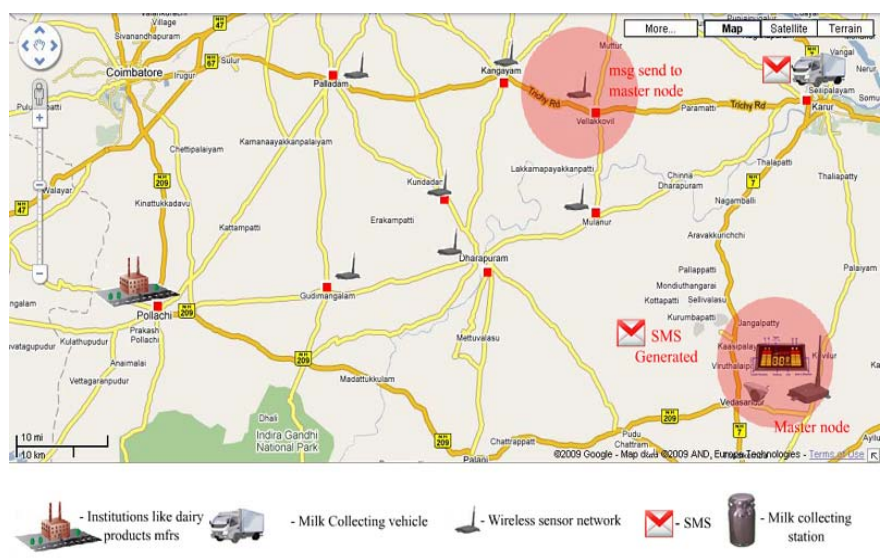

Figure 10: Generation of SMS to the Vehicle Driver

The generated alert SMS will be sent to the vehicle driver which contains the shortest path for milk collection in those stations where the milk may get spoiled soon. 


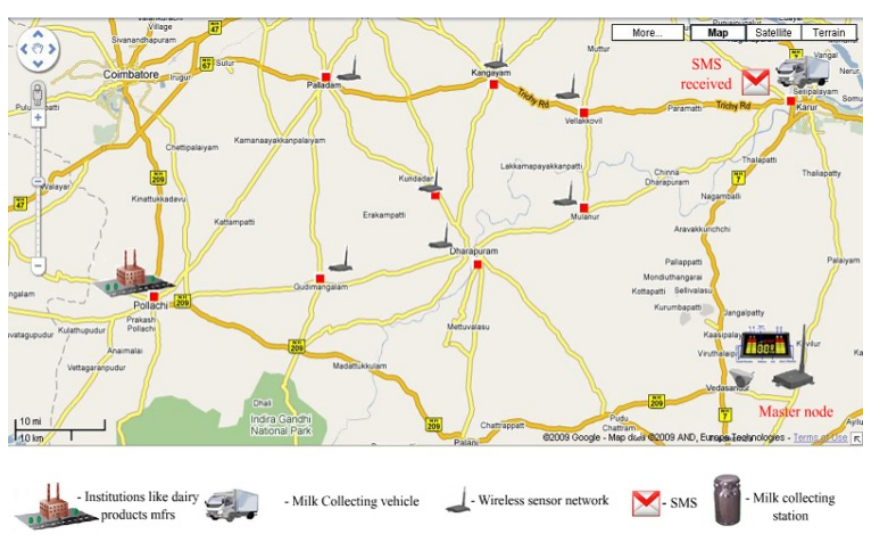

Figure11: Movement of the Vehicle to the Place of Alert generation for the conservation of milk

The vehicle will be travelling in the directed path by the alert SMS and collects the milk before it is getting spoiled.

\section{CONCLUSION}

The conservation of milk is of major concern in developing countries like India, due to the lack of supply at many household in rural and Industrial areas. There is a large amount of spoilage of milk across the country due to the late arrival of vehicle and unavailability of the information at the right time about the condition of milk. Thus the proposed system efficiently conserves the milk and protect from spoilage. As per the cost-benefit analysis, though the installation cost is high, it gives good throughput. We can also extend the proposed model to save any other product to which degradation mainly concentrates on time.

\section{REFERENCES}

[1] F. Baker, "An outsider's view of MANET," Internet Engineering Task Force document, 17 March 2002.

[2] C. Barrett et al., "Characterizing the Interaction between Routing and MAC Protocols in Ad-hoc Networks," Proc. Mobi Hoc 2002, Pp. 92103.

[3] J. Broch et al., "A Performance Comparison of Multi-Hop Wireless Ad Hoc Network Routing Protocols," Proc. Mobicom '98.

[4] D. Cavin et al., "On the accuracy of MANET simulators," Proc. ACM Workshop on Princ. Mobile Computing (POMC'02), Pp. 38-43, Oct. 2002

[5] http://www.dairyforal.com for milk details

[6] http://www.sensor-networks.org/ for wireless sensor networks

[7] http://www.sensormags.com/sensors for sensor details

[8] http://webs.cs.berkeley.edu/tos/dist-1.1.0/tinyos/windows for TinyOS implementation

[9] http://en.wikipedia.org/wiki/ZigBee for ZigBee specification

\section{APPENDIX I}

Blink.nc

configuration Blink \{

\}

implementation \{

components Main, BlinkM, SingleTimer, LedsC;

Main.StdControl-> BlinkM.StdControl;

Main.StdControl-> SingleTimer.StdControl;

BlinkM.Timer->SingleTimer.Timer;
BlinkM.Leds->LedsC; \}

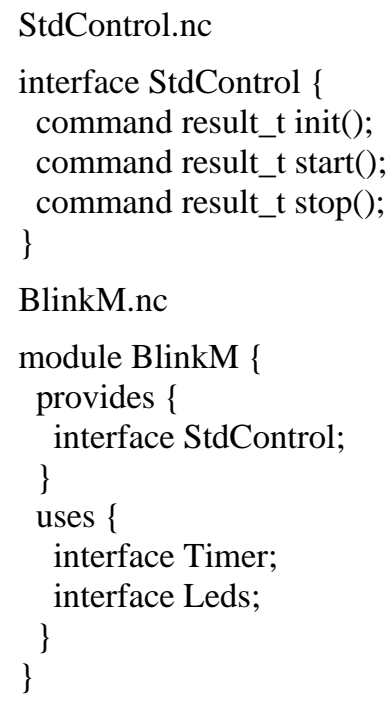

StdControl.nc

interface StdControl \{ command result_t init(); command result_t start(); command result_t stop(); \}

BlinkM.nc

\section{APPENDIX II}

public class SMSClient implements Runnable\{

public final static int SYNCHRONOUS=0; public final static int ASYNCHRONOUS=1; private Thread myThread=null;

private int mode $=-1$;

private String recipient=null;

private String message $=$ null;

public int status=-1;

public long messageNo=-1;

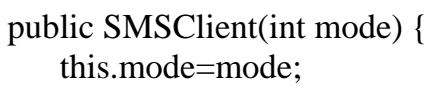

public int sendMessage (String recipient, String message)\{ this.recipient=recipient; this.message $=$ message; //System.out.println("recipient: " + recipient + " message: " + message); myThread = new Thread(this); myThread.start();

// $\operatorname{run}()$; return status; \} public void run()\{

Sender aSender = new Sender(recipient,message);

$\operatorname{try}\{$

//send message aSender.send (); 


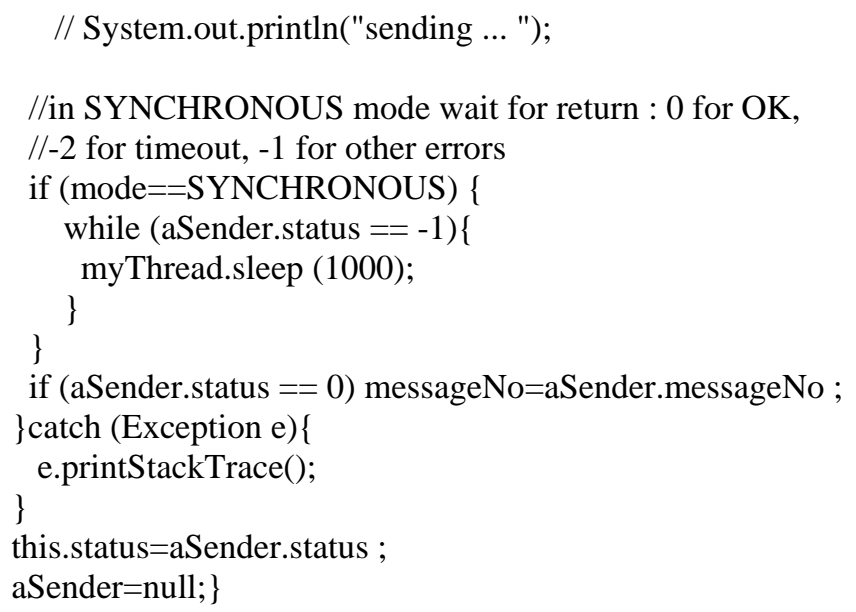

